\title{
Overlapping White Blood Cells Detection Based on Watershed Transform and Circle Fitting
}

\author{
Komal Nain SUKHIA ${ }^{1}$, M. Mohsin RIAZ ${ }^{2}$, Abdul GHAFOOR ${ }^{1}$, Naima ILTAF $^{1}$ \\ ${ }^{1}$ National University of Science and Technology (NUST), Islamabad, Pakistan \\ ${ }^{2}$ COMSATS Institute of Information Technology, Islamabad, Pakistan
}

komalsukhia@students.mcs.edu.pk,mohsin.riaz@comsats.edu.pk, abdulghafoor-mcs@nust.edu.pk, naima@mcs.edu.pk

Submitted February 7, 2017 / Accepted August 9, 2017

\begin{abstract}
White blood cells (WBCs) count and segmentation is considered to be an important step to diagnose diseases like leukemia, malaria etc. Automatic analysis of blood smear images will help hematologists to detect WBCs efficiently and effectively as compared with the manual analysis which is quite time consuming. Therefore, an automatic white blood cells detection technique for complex blood smear images has been proposed. The proposed scheme uses segmentation and edge map extraction for the separation of overlapped WBCs further, parametric circle approximation has been used which is capable of detecting both separated and overlapped white blood cells. Simulation results compared with the existing techniques verify the accuracy and robustness of the proposed scheme.
\end{abstract}

\section{Keywords}

Microscopic imaging, cell detection, circle fitting, segmentation

\section{Introduction}

The automatic analysis of blood smear images for detection of white blood cells (WBCs) has gained significant importance in medical diagnostics. In contrast to manual examination (performed by hematologists), the computerized analysis provides efficiency as it reduces the time complexity and fatigue to a great extent [1], [2]. However, the accuracy of WBC detection is highly dependent on the cell shape, size, texture, color and localization.

WBC detection based on set of features depends on the training samples [2]. Iterative Otsu thresholding detects staining spots along-with WBC [3]. Contrast enhancement (using intensity stretching techniques) and segmentation (based on hue, saturation, intensity model) improve the visibility and decompose the leukemia images into blast and nucleus parts [4]. Unsupervised k-means clustering along with contrast enhancement and the color transformation has been used for segmentation of cytoplasm and nucleus regions [5]. WBC detection has been considered as circle fitting problem in [6] and electromagnetism-like optimization algorithm has been used. The idea is further modified considering it as an ellipse fitting problem optimized using differential evolution (DE) technique [7], [8]. In [11], an imaging system based upon acousto-optic tunable filter has been proposed to detect the WBCs. However, the existing methods [2-8], [11] have limited accuracy in the detection of overlapping cells. In [12], a circle detection algorithm has been used to count red blood cells (RBCs) and WBCs. The method is able to detect a certain degree of overlapping cells but fails to detect highly cluttered cells. In [13], a hybrid system using random forest and fuzzy logic has been proposed to calculate the number of WBCs in blood samples. However, the blood smear images used for the detection purpose contain a single WBC only and multiple overlapped WBCs have not been taken into account. Radial-based cell formation algorithm [14] detects overlapping RBCs and cervical cells but it has not yet been tested on overlapping WBCs in blood smear images. Automatic counting and classification of WBCs based upon morphological features in [15] can not detect irregular and overlapped WBCs accurately. In [16], edge detection and watershed segmentation (WS) have been utilized to separate overlapping WBCs but mostly the separation of overlapping WBCs suffers from over-segmentation even after the application of post-processing steps. The post-processing steps used include erosion and dilation with a structuring element of size 2, which fails to provide accurate segmentation in case of maximum overlapped WBCs. Moreover, RBCs and platelets have also been segmented from the input images for blood count. In [18], blood cells segmentation has been proposed using a variant of watershed transform i.e, minimum area watershed and circle radon transformation (CRT). The proposed method results into over-segmented WBCs and the testing image set does not include overlapped, edge touching cells. In [17], an automated scheme for WBCs analysis from microscopic input images using circular hough transform $(\mathrm{CHT})$ has been proposed. However, in case of maximum overlapped and clustered WBCs, the proposed technique fails to provide accurate count of WBCs. In [19], the abundant information carried by the hyper spectral images has been utilized for WBCs segmentation and classification 
purpose. However, the proposed scheme mainly focuses on the classification of WBCs and consider images containing a single WBC.

An efficient and automatic WBCs detection technique for complex blood smear images has been proposed. The proposed scheme has been based upon the segmentation, edge map extraction and circle fitting algorithm. It is capable of detecting both separated and overlapped WBCs. Simulation results compared with the state-of-the-art techniques verify the accuracy and robustness of proposed scheme.

\section{Proposed Methodology}

Let $X^{(l)}$ be the $l^{\text {th }}$ color band of input image having dimensions $U \times V$, where $l=1,2,3$ represents red, green and blue, $u=1,2, \ldots, U$ and $v=1,2, \ldots, V$ are number of rows and columns respectively. The gray-scale image $X$ has been obtained as

$$
X=0.299 X^{(1)}+0.587 X^{(2)}+0.114 X^{(3)} .
$$

The gray-scale image has been passed through a multilevel Otsu thresholding to obtain two threshold values i.e.,

$$
\left(\xi_{1}, \xi_{2}\right) \underset{\text { (Otsu Thresholding) }}{\text { Multi-level }}(X, 2) .
$$

The main idea of multi-level thresholding is to maximize the inter-class variance function in order to estimate the optimal threshold level for the image. Multi-level Otsu thresholding algorithm helps to identify different clusters (i.e isolating WBCs from the background). An optimal threshold has been computed using the Multi-level Otsu algorithm, which is further utilized to isolate WBCs from the background.

$$
W(u, v)= \begin{cases}0, & \text { if } 0 \leq X(u, v) \leq \xi_{1}, \\ 1, & \text { otherwise. }\end{cases}
$$

The resultant image $W$ is a binary image. Let $\phi_{b}(u, v)$ and $\psi_{b}(u, v)$ represent area and convex area of the $b^{\text {th }}$ connected component located at $(u, v)^{\text {th }}$ location, the binary mask for separating single cells is

$$
S_{1}(u, v)= \begin{cases}1, & \text { if } \phi_{b}(u, v) / \psi_{b}(u, v)>\zeta, \\ 0, & \text { otherwise }\end{cases}
$$

where $\zeta$ is a constant parameter which represents threshold (empirically selected as 0.85). All the connected components having a solidity value greater than a pre-determined threshold have been considered as separated WBCs while the components having solidity value smaller than a predetermined threshold have been regarded as grouped WBCs. The image containing single cell $G_{1}$ is

$$
G_{1}=X \otimes S_{1}
$$

where $\otimes$ is binary AND operator. Similarly the binary mask for separating grouped cells is

$$
S_{2}(u, v)= \begin{cases}1, & \text { if } \phi_{b}(u, v) / \psi_{b}(u, v)<\zeta \\ 0, & \text { otherwise. }\end{cases}
$$

The image containing grouped cell $G_{2}$ is

$$
G_{2}=X \otimes S_{2} .
$$

For the segmentation purpose, marker-controlled watershed transform has been applied on $G_{2}$ to separate the grouped cells. Let $D$ be the distance transform computed using chess board distance and $G_{3}$ be the output image (containing separated cells after watershed transform), i.e.

$$
G_{3} \stackrel{\text { Marker Controlled }}{\text { (Watershed) }}\left(G_{2}, D\right) \text {. }
$$

The $G_{1}$ and $G_{3}$ images have been combined to construct an overall image i.e. $G=G_{1}+G_{3}$. In next step, the edge map has been extracted using morphological operations, i.e.

$$
E=G-(G \ominus \varsigma)
$$

where $\varsigma$ is structuring element and $\ominus$ is erosion operator. Erosion operator (disk shaped structuring element of size 5) has been used to minimize the noise and the non-relevant structures in the resulting image. Edge map image $E$ has been utilized further for circle fitting which is useful to detect overlapped and deformed cells with a complete cell contour. For this purpose, circle fitting algorithm has been used where a circle has been fitted over each WBC and absolute error between edge map and fitted circle has been minimized.

The number of iterations required for circle fitting has not been fixed and it has been considered to be dynamic depending upon the correspondence between the edge map pixels and WBC radius. Mainly iterations depend on two factors i.e. the number of edge pixels in the edge map image for each cell and the distance between the pixels of the candidate circle and the edge map of each cell in each iteration. Step 1: Consider the binary edge map image of each cell and denote the edge map pixels for each WBC as $P_{i}=P_{1}, P_{2}, P_{3}, \ldots, P_{n}$ where $P_{i}$ is the edge map of one WBC.

Step 2: Define a parameter fail which represent the circles which are unfit on the WBC. Initially this parameter is set to be at 0 . The algorithm perform iterations for each WBC edge map pixels i.e. $P_{1}$ to $P_{n}$.

Step 3: Randomly select four edge pixels on the edge map of WBC, from which four candidate circles will be formed. Calculate the distance between the boundary of the candidate circle and edge map pixels for each WBC, which is regarded as circle fitting error. Select that candidate circle as selected circle, which contains highest number of edge map pixels on it's boundary.

Step 4: If none of the candidate circles are fit over the edge map precisely, then fail parameter will be incremented by 1 for each failure case i.e. fail $=$ fail +1 . Then the algorithm proceed towards Step 3 to find other circles.

\section{Results and Discussion}

To evaluate the performance, the proposed and existing DE [8], WS [16], CHT [17], CRT [18] schemes have 


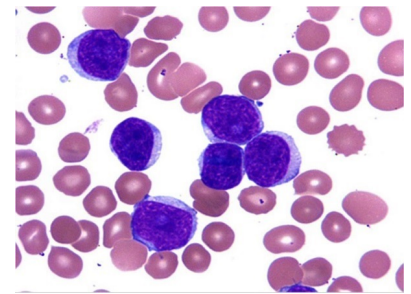

(a)

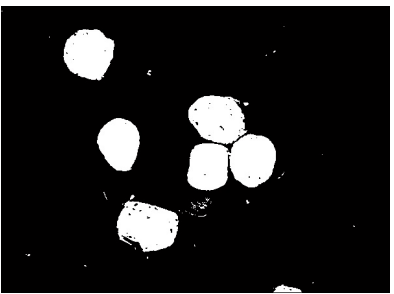

(b)

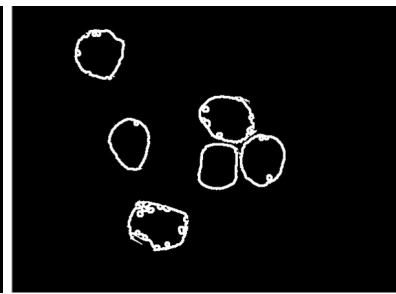

(c)

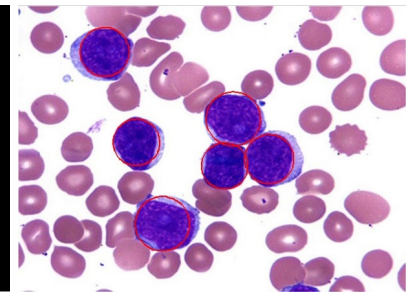

(d)

Fig. 1. Resulting images after applying the proposed scheme (a) Original image, (b) Segmented image, (c) Edge map obtained after erosion, (d) Detected WBCs.

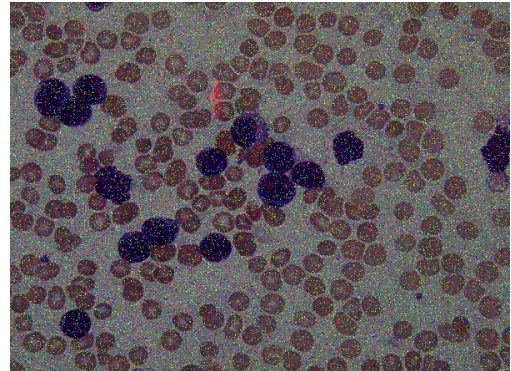

(a)

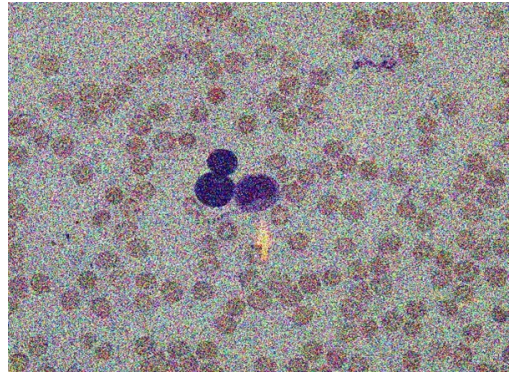

(d)

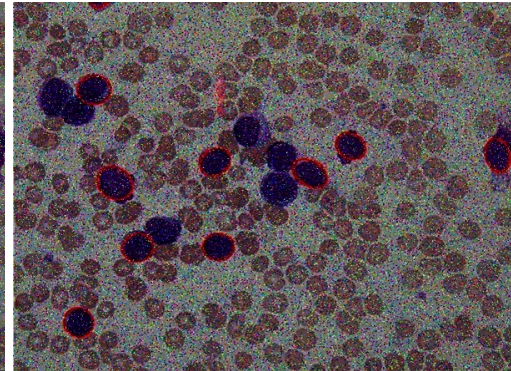

(b)

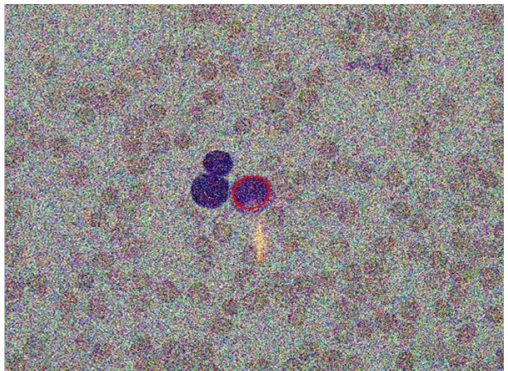

(e)

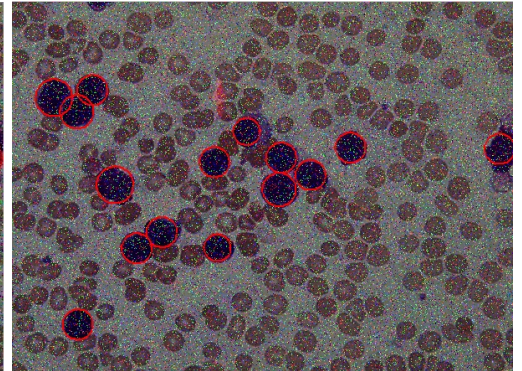

(c)

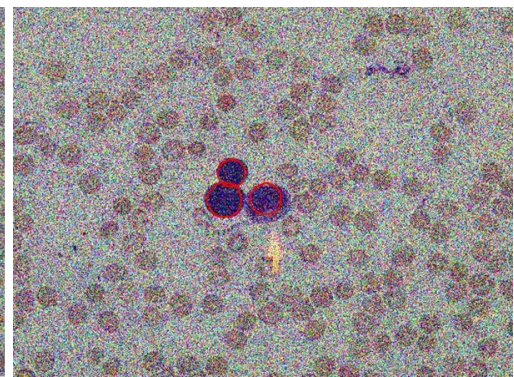

(f)

Fig. 2. WBC detection (a) Corrupted (salt \& pepper $10 \%$ ) image, (b) DE [8] technique, (c) Proposed technique, (d) Corrupted (Gaussian $\sigma=10$ ) noise, (e) DE [8] technique, (f) Proposed technique.

\begin{tabular}{llll}
\hline Noise Type & Technique & DR (\%) & FAR (\%) \\
\hline \multirow{5}{*}{ None } & WS [16] & $82.23 \%$ & $12.00 \%$ \\
& CHT [17] & $75.09 \%$ & $16.66 \%$ \\
& DE [8] & $70.10 \%$ & $13 \%$ \\
& CRT [18] & $78.02 \%$ & $14.12 \%$ \\
& Proposed & $97.10 \%$ & $7.80 \%$ \\
\hline & WS [16] & $79.00 \%$ & $10.22 \%$ \\
Salt \& Pepper (5\%) $)$ & CHT [17] & $68.56 \%$ & $16.78 \%$ \\
& DE [8] & $68.00 \%$ & $11.46 \%$ \\
& CRT [18] & $73.11 \%$ & $15.00 \%$ \\
& Proposed & $96.35 \%$ & $10.42 \%$ \\
\hline & WS [16] & $77.32 \%$ & $13.44 \%$ \\
Salt \& Pepper $(10 \%)$ & CHT [17] & $67.87 \%$ & $18.00 \%$ \\
& DE [8] & $66.67 \%$ & $12.24 \%$ \\
& CRT [18] & $69.67 \%$ & $15.95 \%$ \\
& Proposed & $93.75 \%$ & $11.19 \%$ \\
\hline & WS [16] & $76.33 \%$ & $12.74 \%$ \\
& CHT [17] & $68.17 \%$ & $19.31 \%$ \\
& DE [8] & $68.49 \%$ & $10.15 \%$ \\
Gaussian $(\sigma=5)$ & CRT [18] & $72.22 \%$ & $15.32 \%$ \\
& Proposed & $96.61 \%$ & $9.46 \%$ \\
\hline & WS [16] & $75.72 \%$ & $14.83 \%$ \\
& CHT [17] & $65.95 \%$ & $19.44 \%$ \\
& DE [8] & $67.19 \%$ & $11.19 \%$ \\
& CRT [18] & $67.83 \%$ & $17.23 \%$ \\
& Proposed & $94.53 \%$ & $9.80 \%$ \\
\hline \multirow{5}{*}{ Gaussian $(\sigma=10)$} & &
\end{tabular}

Tab. 1. Quantitative comparison.

been simulated on ALL-IDB [9] and ASH image bank [10] databases. The databases contain a total of $384 \mathrm{WBC}$ images including some overlapping cases. We have mainly focused on the blood smear images having cluttered and overlapping WBCs for the testing purpose. The quantitative comparison has been performed using detection rate (DR) and false alarm rate (FAR). DR has been defined as the ratio of the number of WBCs detected (using proposed or existing scheme) to the number of WBCs counted by the expert (high value of DR is better). FAR has been defined as the ratio of the number of objects which are wrongly detected as WBCs to the number of WBCs counted by the expert (lower value of FAR is better).

Figures 1(a) and 1(b) show original and marker controlled watershed segmented images. Figures 1(c) and 1(d) respectively show the edge map and accurately WBC detected images. It can be viewed that the proposed scheme is capable of detecting WBCs even for complex and overlapping boundaries. The robustness of the proposed scheme has also been verified in the presence of salt $\&$ pepper and Gaussian noises (of different variances/intensities). Figures 2(a) and 2(d) respectively show the original images corrupted 
with salt \& pepper (with $5 \%$ intensity) and Gaussian (with variance $\sigma=10$ ) noises. Note that the DE [8] technique in Fig. 2(b) and 2(e) is unable to detect some WBCs, whereas the proposed scheme in Fig. 2(c) and 2(f) detects almost all the WBCs.

Table 1 shows the quantitative comparison for the existing DE [8], WS [16], CHT [17], CRT [18] and proposed schemes. The comparison with CRT [18] might be unfair since their segmentation mainly focuses on RBCs rather than WBCs. It can be viewed that the proposed approach provides better DR and FAR as compared with DE [8], WS [16], CRT [18] and CHT [17] even in presence of noise.

\section{Conclusion}

This paper has proposed a method to automate the segmentation and counting of WBCs using iterative structured circle fitting algorithm. The proposed scheme mainly uses segmentation and edge map extraction as preprocessing steps. Circle fitting algorithm has been used to detect both separated and overlapped WBCs. Simulation results compared with the existing techniques verify the accuracy and robustness of proposed scheme.

\section{References}

[1] AGAiAn, S., MADHUKAR, M., CHRONOPOUlOS, A. T. Automated screening system for acute myelogenous leukemia detection in blood microscopic images. IEEE Systems Journal, 2014, vol. 8, no. 3, p. 995-1004. DOI: 10.1109/JSYST.2014.2308452

[2] RAMOSER, H., LAURAIN, V., BISCHOF, H., et al. Leukocyte segmentation and classification in blood-smear images. In Proceedings of the International Conference on Engineering in Medicine and Biology Society. 2006, p. 3371-3374. DOI: 10.1109/IEMBS.2005.1617200

[3] WU, J., ZENG, P., ZHOU, Y., et al. A novel color image segmentation method and its application to white blood cell image analysis. In Proceedings of the International Conference on Signal Processing. 2006, p. 1-4. DOI: 10.1109/ICOSP.2006.345700

[4] SALIHAH, A., MASHOR, M. Y., HARUN, N. H., et al. Improving colour image segmentation on acute myelogenous leukaemia images using contrast enhancement techniques. In Proceedings of the IEEE Conference on Biomedical Engineering and Sciences. 2010, p. 246-251. DOI: 10.1109/IECBES.2010.5742237

[5] NASIR, A. S., MASHOR, M. Y., ROSLINE, H. Unsupervised colour segmentation of white blood cell for acute leukemia images. In Proceedings of the International Conference on Imaging Systems and Techniques. 2011, p. 1-4. DOI: 10.1109/IST.2011.5962188

[6] CUEVAS, E., OLIVA, D., DÍAZ, M., et al. White blood cell segmentation by circle detection using electromagnetism-like optimization. Computational and Mathematical Methods in Medicine, 2013, p. 1-15. DOI: $10.1155 / 2013 / 395071$

[7] SARASWAT, M., ARYA, K. V., SHARMA, H. Leukocyte segmentation in tissue images using differential evolution algorithm. Swarm and Evolutionary Computation, 2013, vol. 11, p. 46-54. DOI: $10.1016 /$ j.swevo.2013.02.003

[8] CUEVAS, E., DÍAZ, M., MANZANARES, M., et al. An improved computer vision method for white blood cells detection. Computational and Mathematical Methods in Medicine, 2013, p. 1-14. DOI: $10.1155 / 2013 / 137392$
[9] LABATI, R. D., PIURI, V., SCOTTI, F. ALL-IDB: The acute lymphoblastic leukemia image database for image processing. In Proceedings of the IEEE International Conference on Image Processing. 2011, p. 2045-2048. DOI: 10.1109/ICIP.2011.6115881

[10] ASH IMAGE BANK, AMERICAN SOCIETY OF HEMATOLOGY, USA. [Online] Cited 2017-02-07. Available at: http://imagebank.hematology.org/Default.aspx

[11] LI, Q., WANG, Y., LIU, H., et al. Leukocyte cells identification and quantitative morphometry based on molecular hyperspectral imaging technology. Computerized Medical Imaging and Graphics, 2014, vol. 38, no. 3, p. 171-178. DOI: 10.1016/j.compmedimag.2013.12.008

[12] AlOMARI, Y. M., ABDUllah, S. N. H. S., AZMA, R. Z., et al. Automatic detection and quantification of WBCs and RBCs using iterative structured circle detection algorithm. Computational and Mathematical Methods in Medicine, 2014, p. 1-17. DOI: $10.1155 / 2014 / 979302$

[13] GHOSH, P., BHATTACHARJEE, D., NASIPURI, M. Blood smear analyzer for white blood cell counting: A hybridmicroscopic image analyzing technique. Applied Soft Computing, 2015, vol. 46, p. 629-638. DOI: 10.1016/j.asoc.2015.12.038

[14] KANAFIAH, S. N. A. M., JUSMAN, Y., ISA, N. A. M., et al. Radial-based cell formation algorithm for separation of overlapping cells in medical microscopic images. In International Conference on Computer Science and Computational Intelligence. 2015, vol. 59, p. 123-132. DOI: $10.1016 /$ j.procs.2015.07.522

[15] NAZLIBILEK, S., KARACOR, D., ERCAN, T., et al. Automatic segmentation, counting, size determination and classification of white blood cells. Measurement, 2014, vol. 55, p. 58-65. DOI: 10.1016/j.measurement.2014.04.008

[16] SAVKARE, S. S., NAROTE, S. Blood cell segmentation from microscopic blood images. In IEEE International Conference on Information Processing. 2015, p. 502-505. DOI: 10.1109/INFOP.2015.7489435

[17] PAVITHRA, S., BAGYAMANI, J. White blood cell analysis using watershed and circular hough transform technique. International Journal of Computational Intelligence and Informatics, 2015, vol. 5, no. 2, p. 114-123. ISSN: 2349-6363.

[18] TEK, F. B., DEMPSTER, A. G., KALE, I. Blood cell segmentation using minimum area watershed and circle radon transformations. Mathematical Morphology, 2005, p. 441-454. DOI: $10.1007 / 1-4020-3443-1 \_40$

[19] WANG, Q., CHANG, L., ZHOU, M., et al. A spectral and morphologic method for white blood cell classification. Optics and Laser Technology, 2016, vol. 84, p. 144-148. DOI: 10.1016/j.optlastec.2016.05.013

\section{About the Authors...}

Komal Nain SUKHIA has obtained her MS degree in 2015 from National University of Sciences and Technology, Pakistan. Currently, she is pursuing her Ph.D. in same institute and actively involved in research activities related to image processing.

M. Mohsin RIAZ obtained his Ph.D. Electrical Engineering in 2013 from National University of Sciences and Technology, Pakistan. Currently, he is serving at COMSATS Institute of Information Technology, Pakistan. His research interests include signal and image processing, pattern recognition, evolutionary computing and fuzzy logic. 
Abdul GHAFOOR obtained his Ph.D. Control Systems in 2008 from University of Western Australia. Currently, he is Head of Information Security department at National University of Sciences and Technology, Pakistan. His research interests include model and controller reduction, image processing (enhancement, fusion, segmentation and watermarking), cognitive radios and ground penetration radar.
Naima ILTAF obtained her Ph.D. Software Engineering in 2013 from National University of Sciences and Technology, Pakistan. Currently, she is serving National University of Sciences and Technology, Pakistan. Her research interests include image processing, machine learning, trust and recommender system. 